\title{
Identification and removal of reflection artifacts in minimally invasive photoacoustic imaging for accurate visualization of brachytherapy seeds
}

Mithun Kuniyil Ajith Singh, Vinay Parameshwarappa, Ellen Hendriksen, Wiendelt Steenbergen, Srirang Manohar

Mithun Kuniyil Ajith Singh, Vinay Parameshwarappa, Ellen Hendriksen, Wiendelt Steenbergen, Srirang Manohar, "Identification and removal of reflection artifacts in minimally invasive photoacoustic imaging for accurate visualization of brachytherapy seeds," Proc. SPIE 10064, Photons Plus Ultrasound: Imaging and Sensing 2017, 100640G (23 March 2017); doi: $10.1117 / 12.2252561$ 


\title{
Identification and removal of reflection artifacts in minimally-invasive photoacoustic imaging for accurate visualization of brachytherapy seeds
}

\author{
Mithun Kuniyil Ajith Singh ${ }^{\mathrm{a} *}$, Vinay Parameshwarappa ${ }^{\mathrm{a}}$, Ellen Hendriksen ${ }^{\mathrm{b}}$, Wiendelt Steenbergen ${ }^{\mathrm{a}}$, \\ Srirang Manohar ${ }^{\mathrm{a}}$ \\ ${ }^{a}$ Biomedical Photonic Imaging Group, MIRA Institute for Biomedical Technology and Technical \\ Medicine, University of Twente, P.O. Box 217, 7500 AE Enschede, The Netherlands \\ ${ }^{b}$ Department of Radiation Oncology, Medisch Spectrum Twente, P.O. Box 50000, 7512 KZ \\ Enschede, The Netherlands \\ ‥kuniyilajithsingh@utwente.nl
}

\begin{abstract}
Reflection artifacts caused by the high signal from the optical fiber/ needle tip reflecting off the seed is an important problem in minimally invasive photoacoustic imaging of brachytherapy seeds. The presence of these artifacts confounds the interpretation of images and reduces contrast. We apply a new method called PAFUSion (Photoacoustic-guided focused ultrasound) to identify and reduce reflection artifacts generated in interstitial illumination imaging of brachytherapy seeds. We present the system comprising of a US imager and linear array, with illumination provided via a cutting needle. Non-radioactive brachytherapy seeds are implanted in a tissue mimicking phantom and ex vivo porcine tissue. The PAFUSion-corrected imaging results successfully demonstrate that our approach can identify and strongly reduce reflection artifacts in the context of photoacoustic needle. The phantom result also shows that multi-spectral photoacoustics can separate signals between the seeds and other optical absorbers.
\end{abstract}

Keywords: Photoacoustics, Ultrasound, Reflection artifacts, Photoacoustic needle, Photoacoustic-guided focused ultrasound, Brachytherapy

\section{INTRODUCTION}

Prostate cancer is the leading cause of cancer deaths among men. ${ }^{1}$ The most standard treatment approaches for prostate cancer include radiotherapy and radical prostatectomy. Radiotherapy is a form of treatment where the control of disease is performed by administrating high radioactive dose either superficially or interstitially. Brachytherapy is a targeted radiation therapy treatment which enables high-precision delivery of radiation doses to the tumor area in the prostate. ${ }^{2}$ In brachytherapy, the localized tumor area instead of the whole prostate is targeted with defined radiation dose, using multiple (50 to 100) tiny metallic radioactive sources implanted inside the prostate.

The main purpose of brachytherapy seed implantation is to deliver radiation dose to the cancer bearing prostate and hence avoiding radiation exposure to the healthy tissue. For attaining a planned radiation dose to the tumorous tissue, brachytherapy is usually performed by following a treatment plan. Deviation between planned and delivered radiation dose may lead to complications. ${ }^{3}$ Mismatch between the planned and delivered radiation dose can be avoided to a large extent by accurately visualizing the seeds during the implantation procedure and making sure that the seeds are placed at right places based on the treatment plan. ${ }^{2}$ TRUS (Trans-rectal ultrasound) imaging is commonly used for real-time guidance for seed implantation and visualization inside the prostate during brachytherapy treatment. However, seeds are often challenging to visualize using ultrasound (US) due to their small size, their similarity in acoustic impedance with microcalcifications, and due to the presence of characteristic acoustic artifacts such as shadowing and reverberations. ${ }^{4}$

To overcome the shortcomings of US imaging for visualizing the seeds intraoperatively, photoacoustic (PA) imaging can be used. ${ }^{4,5,6}$ PA imaging has the potential to complement US detection of brachytherapy seeds. In PA imaging, pulsed light absorbed by the target emits US by thermoelastic mechanism. ${ }^{7}$ This US is detected using a US detector array to build up an image. PA imaging offers spectroscopic optical absorption contrast with US resolution. ${ }^{8}$ Owing to the high optical absorption of metal compared to the tissue, PA imaging offers excellent contrast in imaging brachytherapy seeds. ${ }^{4,5,6}$ Considering the imaging depth required, the PA needle ${ }^{9,10}$ with interstitial light delivery via a cutting percutaneous needle (Fig. 1a) is generating much interest in imaging brachytherapy seeds. The challenge in interstitial

Photons Plus Ultrasound: Imaging and Sensing 2017, edited by Alexander A. Oraevsky, Lihong V. Wang, Proc. of SPIE Vol. 10064, 100640G · @ 2017 SPIE · CCC code: 1605-7422/17/\$18 · doi: $10.1117 / 12.2252561$

Proc. of SPIE Vol. 10064 100640G-1 
PA imaging of brachytherapy seeds is the presence of reflection artifacts caused by the high PA signal from the fiber/needle tip reflecting off the seeds (Fig. 1a). ${ }^{6}$ These artifacts may appear in the region of interest and can reduce the contrast and cause difficulty in image interpretation as reflection artifacts can be misinterpreted as seeds. ${ }^{6}$ It is thus important to identify and reduce reflection artifacts for accurate interstitial PA visualization of brachytherapy seeds.

We recently proposed a method called photoacoustic-guided focused ultrasound (PAFUSion) to identify reflection artifacts in epi-mode PA imaging. ${ }^{11}$ In this work, we study the use of PAFUSion in identifying and reducing in-plane reflection artifacts with the use of the PA needle in the visualization of brachytherapy seeds. We present the results of the combined approach in visualizing non-radioactive brachytherapy seeds in a tissue mimicking phantom and an $e x$ vivo porcine tissue. In addition, we also show the potential of using multispectral PA for separating seed signals from other optical absorbers like blood in a phantom measurement.

\section{METHODOLOGY AND MATERIALS}

\subsection{PAFUSion-enabled PA needle for accurate visualization of brachytherapy seeds - Idea}

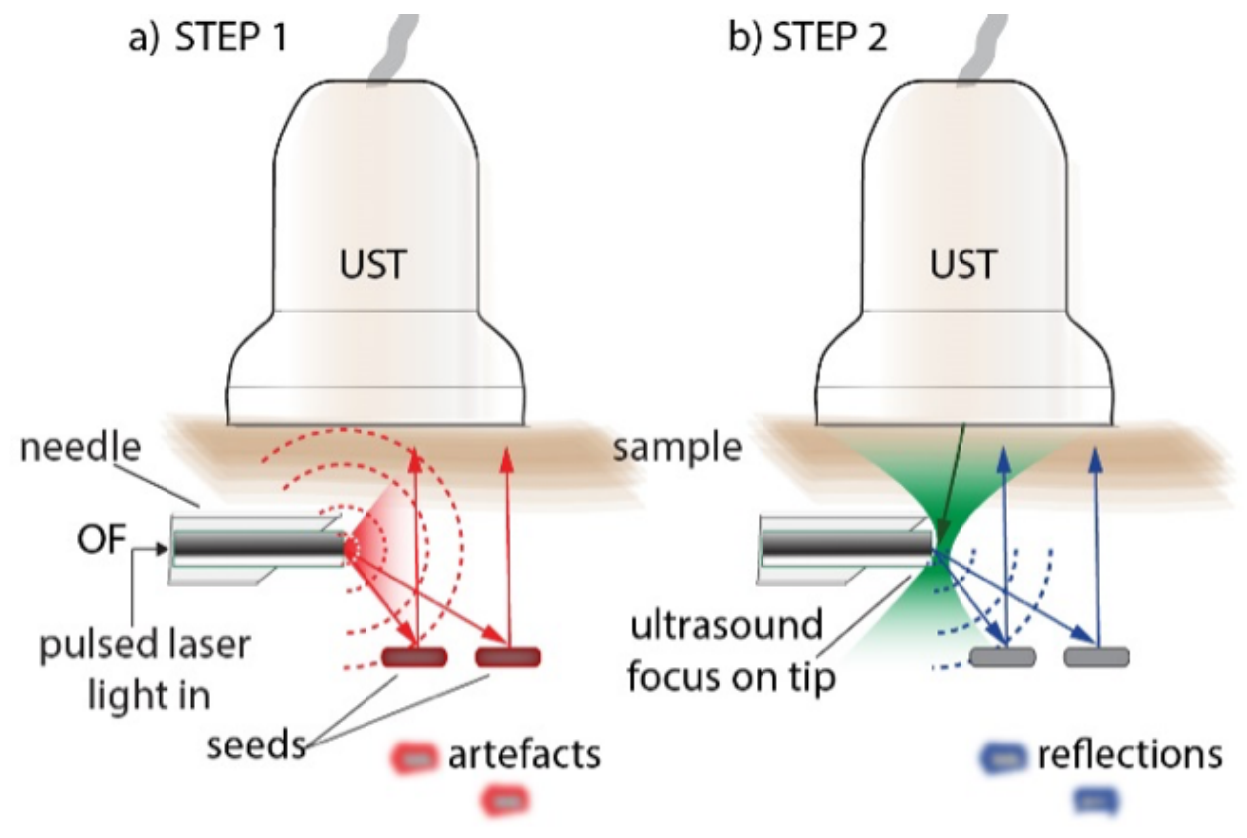

Figure 1: (a) Concept of the Photoacoustic needle, where pulsed light is applied in tissue via an optical fiber (OF) in a cutting needle with external ultrasound detection. By this, tissue at the optical fiber tip, the tip itself and the brachytherapy seeds can be visualized. Additionally, however artifacts are also visualized due to reflection on the seeds of the high photoacoustic signal from the fiber/needle tip. (b) Concept of PAFUSion where in a second step, ultrasound from the transducer is focused on needle/fiber-tip. The ultrasound diverged from the tip is in a first approximation a mimic of the photoacoustic signal from tip in the first step. The detected signals visualize only the reflections from seed and other inhomogeneities allowing identification of the reflection artifacts of the first step.

PAFUSion in general uses US pulses to mimic the wave-field produced by PA sources and thus provides a way to identify reflection artifacts in clinical combined PA and pulse-echo US. ${ }^{11,12}$ In this work, we study the use of PAFUSion in identifying and reducing reflection artifacts with the use of PA-needle in the visualization of brachytherapy seeds. The PAFUSion approach specifically in the context of the photoacoustic needle is shown in Fig. 1a and b. Once the needle tip has been identified from the PA image in step 1 (Fig. 1a), US is focused to this point in a second step. The resultant US data processed using the PAFUSion algorithm ${ }^{11}$ generates an image with only reflections from deeper structures (PAFUSion image) hence representing the artifacts in the PA image from step 1. The average image pixel value of the reflection artifact with the highest image value is then scaled to the image value of its counterpart in the PA image, and the same scaling is applied to all other identified artifacts in the PAFUSion image. After this, reconstructed and 
enveloped PAFUSion data is subtracted from the PA data and the positive parts of the result are plotted to obtain the corrected PA image. The negative parts of the result are assigned an image value of zero.

\subsection{Equipment and setup}

Figure 2 shows the block diagram of the real-time photoacoustic needle imaging system setup. As shown in the figure, the system consists of a commercial handheld US imager (MyLab_One, Esaote Europe BV, Maastricht, the Netherlands) with a linear array probe SL3323 (128 elements, $7.5 \mathrm{MHz}, 100 \%$ bandwidth). ${ }^{13}$ The US system was used in the research mode which was connected to a PC installed with custom made software for controlling US transmission, laser pulse transmission and data acquisition. Nano-second laser pulse light illumination was provided by an ND:YAG laser (Quanta-Ray pro 250, Spectra Physics, Mountain View, California) pumping an optical parametric oscillator (OPO, VeraScan-L532, GWU, Erftstadt, Germany) at $10 \mathrm{~Hz}$. The energy per pulse was monitored using a photodetector (Model 1621, Si, 350-1000nm, $0.8 \mathrm{~mm}$ Diameter, 8-32/ M4, Newport Corporation, California). A multimode optical fiber having a numerical aperture of 0.22 and a diameter of $600 \mu \mathrm{m}$ is coupled to the laser. The proximal and the distal flat ends of the optical fiber are polished to remove any surface roughness and provide better coupling and light transmission. The proximal part of the fiber is coupled to the laser and the distal part of the fiber is held in a core biopsy metal needle. The PA frame rate is $10 \mathrm{~Hz}$ and images can be displayed online using a GPU based delay and sum reconstruction algorithm. Images presented here are averaged over 100 frames and reconstructed offline using a frequency domain reconstruction algorithm ${ }^{14}$, assuming constant speed of sound in the medium for all measurements (phantom measurement: $1480 \mathrm{~m} / \mathrm{s}$, ex-vivo measurement: $1540 \mathrm{~m} / \mathrm{s}$ ).

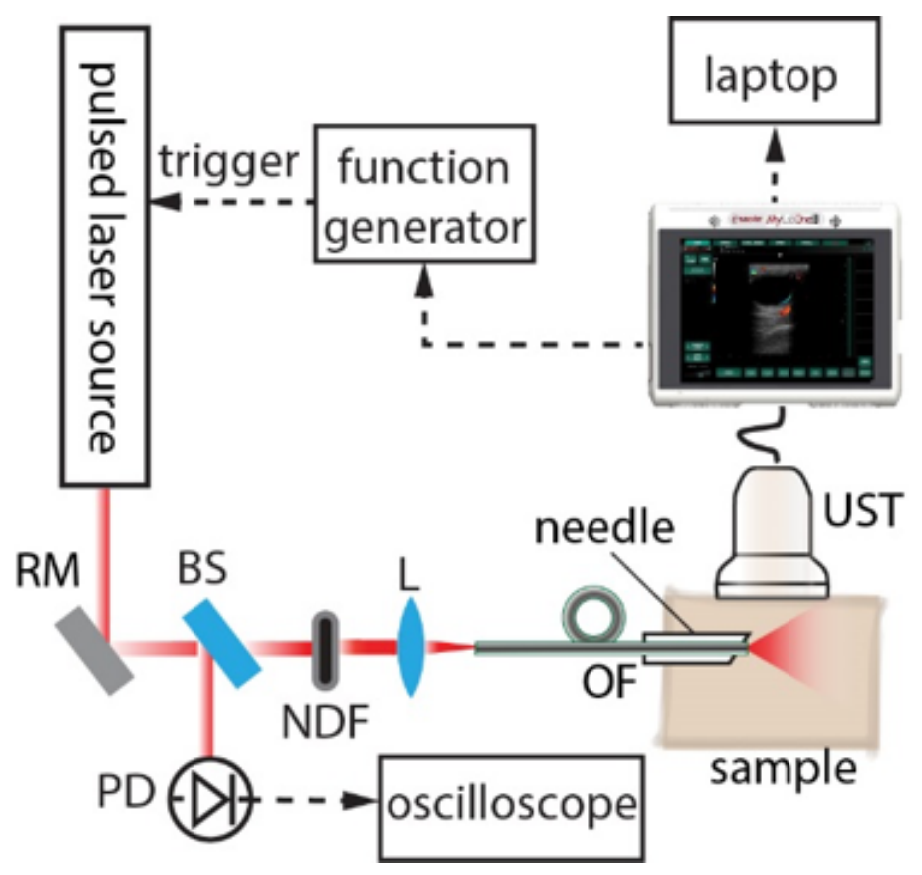

Figure 2: Illustration of setup. RM: reflecting mirror; BS: beam splitter; photodetector; NDF: neutral density filter; L: lens; OF: optical fiber; UST: ultrasound transducer.

\subsection{Phantom experiment}

Fig. 3a shows the schematic of the phantom used for the validation of PAFUSion-enabled PA needle for accurately visualizing brachytherapy seeds. This phantom was prepared with 3\% Agar dissolved in an aqueous solution of Intralipid (I141, 20\% emulsion, Sigma Aldrich) to provide a bulk reduced scattering coefficient of $\mu_{s}^{\prime}=1 \mathrm{~mm}^{-1}$. Three nonradioactive brachytherapy seeds were embedded in the phantom. The seeds were coated with a single layer of India ink to increase optical absorption. A single bead ( $2.2 \mathrm{~mm}$ diameter) of sodium alginate carrying gold nanorods (AuNR) was also embedded in the phantom. The AuNR bead was embedded between the seed 1 and seed 2 . The AuNR were prepared 
using a well-known synthesis protocol. ${ }^{15}$ The reference absorption spectrum of the bead shows an absorption peak at 795 $\mathrm{nm}$ (Fig. 3d). Water coupling was used in between the US probe and the phantom. After acquiring PA and US images, PAFUSion data was collected as performed in the non-scattering phantom experiment. PA imaging was done at a wavelength of $800 \mathrm{~nm}$ with pulse energy of $1.2 \mathrm{~mJ}$. For multi- spectral analysis, PA imaging was carried out at different excitation wavelengths ranging from $720 \mathrm{~nm}$ to $860 \mathrm{~nm}$ in $5 \mathrm{~nm}$ increments.

\subsection{Ex vivo measurements}

Ex vivo validation of the approach was performed using four brachytherapy seeds (coated with single layer of black ink) embedded inside porcine tissue. Using US guidance, the seeds were implanted inside the tissue using a standard $18 \mathrm{G}$ brachytherapy needle (Bard, Brachystar, Covington, Georgia). Orientation of the needle and arrangement of seeds inside the porcine tissue are schematically shown in Fig. 4a. For better acoustic coupling, the sample was covered with thin transparent polyethylene sheet $(\sim 20 \mu \mathrm{m})$ after smearing with US gel (Fig. 4d). The same data acquisition and processing protocol was followed as for the phantom experiment.

\section{RESULTS}

\subsection{Phantom experiment}
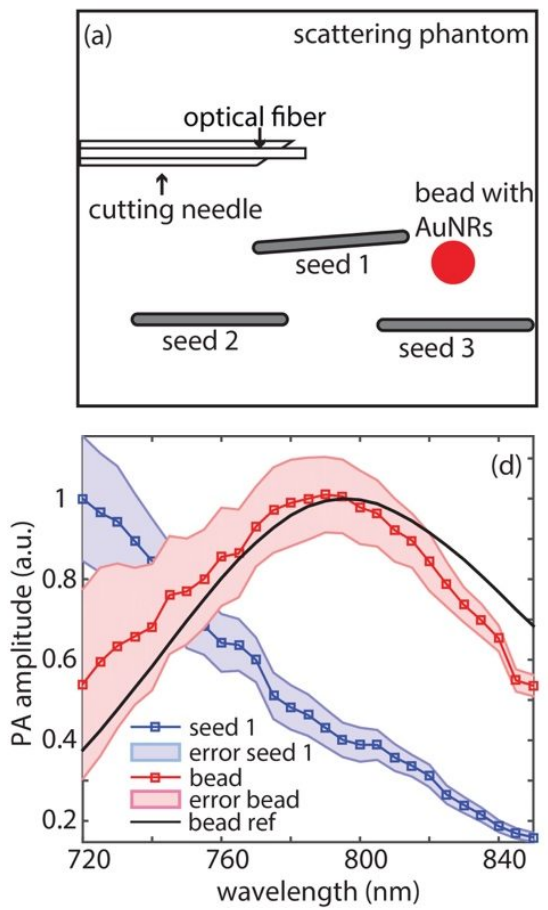
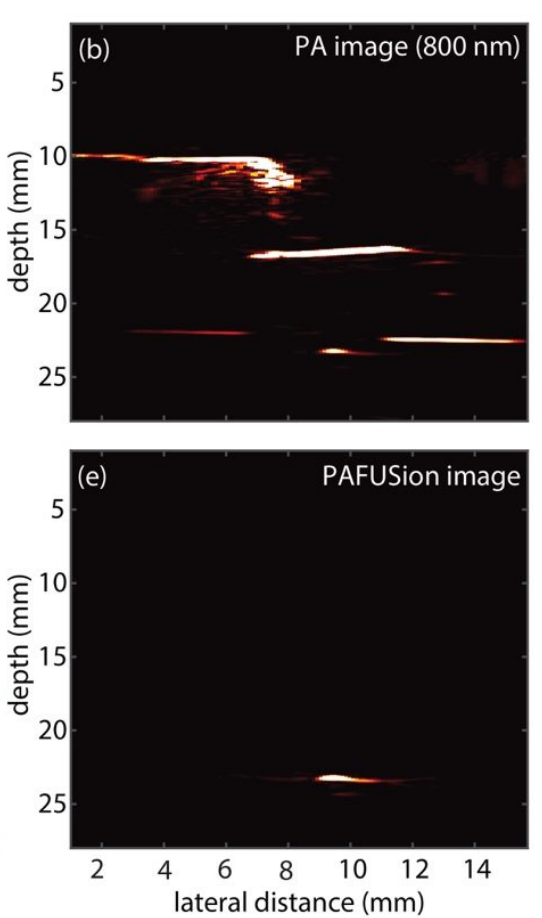
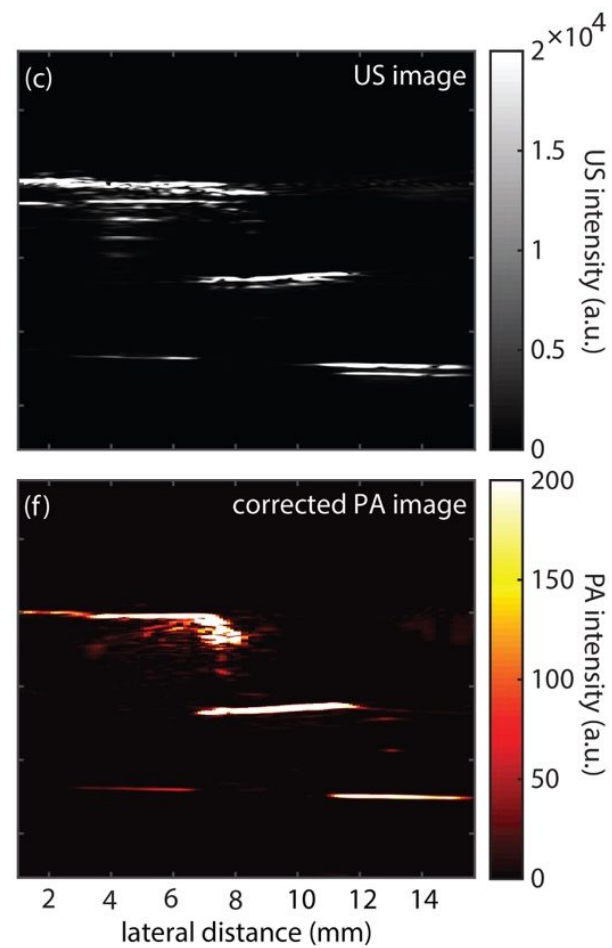

Figure 3: (a) Schematic of the experiment showing orientation of the PA needle, arrangement of seeds and AuNR bead in the Agar phantom with Intralipid, (b) PA image at a wavelength of $800 \mathrm{~nm}$ showing the expected features and with an additional feature at 9.5

$\times 24 \mathrm{~mm} 2$ (lateral distance $\times$ depth), (c) plane- wave US image, (d) measured PA spectrum of seed 1, bead with error margins (standard deviation of the averaged mean) compared with reference spectrum of the bead, (e) PAFUSion image showing that the unknown feature is a reflection artifact, and (f) corrected PA image obtained by subtracting scaled PAFUSion image from the PA image.

Figure $3 \mathrm{~b}$ shows the reconstructed PA image (phantom experiment) in which the shaft and the fiber/needle tip and all three seeds are visualized. The AuNR bead is seen only with top and bottom surfaces, as expected in a limited-view situation. The US image (Fig. 3c) shows the needle shaft and the seeds. In an ideal case, only the optically absorbing structures are expected to show up in the reconstructed PA image. However, a further feature is visible in the PA image 
(lateral distance $=9.5 \mathrm{~mm}$, depth $=24 \mathrm{~mm}$ ) which could be interpreted as an optically absorbing feature, e.g., another seed. The PAFUSion image (Fig. 3e) reveals this feature as a reflection artifact. Figure $3 \mathrm{f}$ shows the corrected PA image obtained by subtracting the PAFUSion-identified reflection artifacts from the real PA image showing a reduction of the artifact. The image values of real PA features are unaffected in the corrected PA image. In Fig. 3d, the PA image values of seed 1 and that of the AuNR bead (normalized to the measured pulse energy) are plotted against the wavelength, together with the reference spectrum of AuNR. The image values of ink-coated seeds decrease monotonically with wavelength, while the PA spectrum of the bead shows the signature longitudinal plasmon peak at roughly $795 \mathrm{~nm}$.

\subsection{Ex vivo measurement}
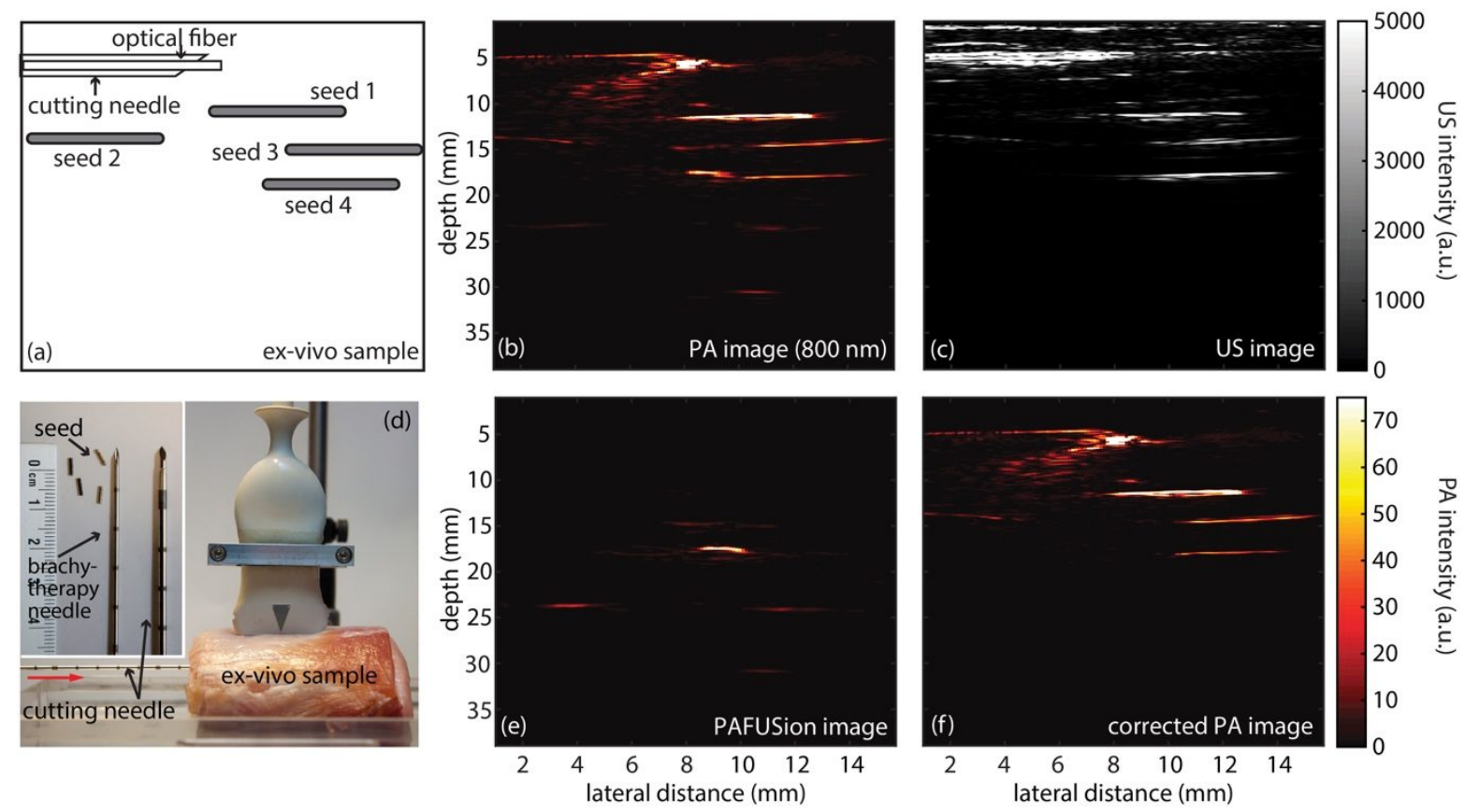

Figure 4: (a) Schematic showing orientation of the PA needle and arrangement of seeds inside the porcine tissue, (b) PA image at a wavelength of $800 \mathrm{~nm}$, (c) plane-wave US image, (d) photograph of the imaging situation with needle inserted in the ex vivo sample. Inset is a photograph of the inner and outer needles alongside the seed. (e) PAFUSion image with the identified reflection artifacts, and (f) corrected PA image obtained by subtracting the scaled PAFUSion image from the PA image.

Figure 4a shows the schematic of the arrangement of seeds inside the ex vivo pork tissue and the orientation of PA needle in the measurement setup. Figure $4 \mathrm{~b}$ shows the reconstructed PA image in which PA fiber/needle tip, all four seeds, and several other bright features are visible. The seed 1 was positioned at an axial distance of $5 \mathrm{~mm}$ with respect to the needle. The measured axial distance between seed 3 and seed 1 is $4.2 \mathrm{~mm}$ whereas the distance between seed 2 and seed 1 was $4.1 \mathrm{~mm}$. Seed 2 appears slightly angled which may have been caused during seed insertion inside the tissue sample. Several additional features with shapes similar to the seeds are also visible. These features are located at different depths which do not correspond to the actual seed locations. These features create difficulty in interpreting the image, for example when counting the number of seeds that are implanted. These additional features can be misinterpreted as seeds, hence causing error in seed count and affecting the radiation dose delivered to the prostate in a clinical scenario.

Figure 4c shows the reconstructed US image. The shaft of the PA needle, all the four seeds and several other echogenic tissue structures are visible in the US image. Figure 4e shows the PAFUSion image from which reflection artifacts in the PA image can be identified. The PAFUSion image shows that a number of features in the PA image are reflection artifacts. All of them are caused by fiber/needle tip PA signal reflecting off the seeds, and off other tissue echogenic structures. Figure $4 \mathrm{f}$ shows the corrected PA image obtained by subtracting the PAFUSion-identified reflection artifacts from the real PA image. It is evident that the intensity of the reflection artifacts are significantly reduced by using PAFUSion without affecting the intensity of real PA features. An interesting additional feature is visible at the same 
depth of seed 3 in the PA image (lateral distance $=7.5 \mathrm{~mm}$, depth $=15 \mathrm{~mm}$ ). The PAFUSion image uncovered this feature as a reflection artifact. This would have been caused by the fiber/needle tip signal reflecting off the point-like tissue feature visible just above the seed 1 (lateral distance $=7.5 \mathrm{~mm}$, depth $=10 \mathrm{~mm}$ ) in both the PA and US image.

\section{DISCUSSION}

Our phantom and ex vivo results demonstrate that PAFUSion-enabled PA needle can accurately visualize brachytherapy seeds. Reflection artifacts present a challenge for photoacoustic needle imaging of brachytherapy seeds, and clinical translations to humans. In both our experiments, it is clear that our approach can eliminate the reflection artifacts caused by the high PA signal from the optical fiber/needle-tip. The method has been developed in the context of PA-needle imaging of brachytherapy seeds, but is potentially suited for all applications of the PA-needle where reflection artifacts could result due to the presence of acoustic reflectors.

Accurate identification of the optical fiber/needle-tip is critical for our approach to work. However, synthetic backpropagation of PA signals (from the fiber/needle-tip) instead of physical focusing may be a solution for this. Matching the shapes of actual reflection artifacts to the PAFUSion-identified artifacts using software filters ${ }^{12}$ may potentially improve the image subtraction without degrading the contrast of real PA features in close vicinity to the reflection artifacts. PAFUSion will be able to identify and reduce reflection artifacts generated inside the imaging plane only. If the fiber/needle-tip is outside the imaging plane, it will be impossible to reduce reflection artifacts using our approach. However, in-plane needle insertion is commonly used in clinical practice for accurate guidance of the needle tip, making this less a limitation.

Angular distribution of ultrasonic wavefield after the focus depth is an important parameter when using PAFUSion in the context of PA needle, as this angle defines the field-of-view of the method in identifying and eliminating reflection artifacts. The angular distribution of US after the focus depth is equal to the angular aperture of the transmitted focused pulse. The latter is limited by the angle of US transmission at which the transmission phase difference between neighboring elements results in destructive interference. In our case where center frequency was $7.5 \mathrm{MHz}$ and the pitch was $0.245 \mathrm{~mm}$, this angle limit was at $+-24^{\circ}$, and was sufficient to identify the reflection artifacts in our study.

Coating the needle tip with a dye/paint with a spectral signature and identifying the reflection artifacts caused by it using spectral decomposition and correcting for it will potentially be an alternate solution for the reflection artifact reduction in interstitial PA imaging. However, this requires further investigation.

\section{CONCLUSIONS}

PAFUSion enables identification and substantial reduction of reflection artifacts when using PA-needle, considerably improving the visualization of brachytherapy seeds. The phantom results also confirm that multi-spectral PA can be potentially a useful tool to separate signals from the brachytherapy seeds and other optical absorbers in the tissue. In a future clinical scenario, we envisage that the PA needle in combination with real-time PAFUSion will provide accurate visualization of the distribution of brachytherapy seeds, which in turn can provide feedback to the radiotherapist regarding adherence to the therapy plan.

\section{ACKNOWLEDGEMENTS}

M.K.A.S. and W.S. are funded by the European Community's Seventh Framework Programme (FP7/2007-2013) under grant Agreement No. 318067. S.M. is funded by the PIHC-Pioneers in Healthcare Innovation funds 2014, and the FP7 program INDIGO Policy in project RTQ-PAI. Authors acknowledge Dr. Altaf Hussain and Johan van Hespen for their insightful discussions and help in experiments. Dr. Peter Brands and engineers from ESAOTE Europe BV are thanked for discussions and support with the MyLab_One system.

\section{REFERENCES}

[1] J. Crook, "The role of brachytherapy in the definitive management of prostate cancer," Cancer Radiother 15(3), 230-237 (2011).

[2] A. J. Chang et al., "High-risk prostate cancer-classification and therapy," Nature Reviews Clinical Oncology 11(6), 308-323 (2014). 
[3] E. M. Messing et al., "Intraoperative optimized inverse planning for prostate brachytherapy: early experience," International journal of radiation oncology, biology, physics 44(4), 801-808 (1999).

[4] J. L. Su et al., "Photoacoustic imaging of prostate brachytherapy seeds," Biomedical optics express 2(8), 22432254 (2011).

[5] T. Harrison, and R. J. Zemp, "Coregistered photoacoustic-ultrasound imaging applied to brachytherapy," Journal of biomedical optics 16(8), (2011).

[6] M. A. L. Bell et al., "In vivo visualization of prostate brachytherapy seeds with photoacoustic imaging," Journal of biomedical optics 19(12), (2014).

[7] Y. Zhou, J. Yao, and L. V. Wang, "Tutorial on photoacoustic tomography," Journal of biomedical optics 21(6), 61007 (2016).

[8] M. Kuniyil Ajith Singh, W. Steenbergen, and S. Manohar, "Handheld Probe-Based Dual Mode Ultrasound/Photoacoustics for Biomedical Imaging," in Frontiers in Biophotonics for Translational Medicine M. Olivo, and U. S. Dinish, Eds., pp. 209-247, Springer Singapore (2016).

[9] D. Piras et al., "Photoacoustic needle: minimally invasive guidance to biopsy," Journal of biomedical optics 18(7), $070502(2013)$.

[10] W. Xia et al., "Performance characteristics of an interventional multispectral photoacoustic imaging system for guiding minimally invasive procedures," Journal of biomedical optics 20(8), 86005 (2015).

[11] M. Kuniyil Ajith Singh, and W. Steenbergen, "Photoacoustic-guided focused ultrasound (PAFUSion) for identifying reflection artifacts in photoacoustic imaging," Photoacoustics 3(4), 123-131 (2015).

[12] M. Kuniyil Ajith Singh, M. Jaeger, M. Frenz, and W. Steenbergen, "In vivo demonstration of reflection artifact reduction in photoacoustic imaging using photoacoustic-guided focused ultrasound," Biomedical optics express, 7(8), 2955-2972 (2016).

[13] K. Daoudi et al., "Handheld probe integrating laser diode and ultrasound transducer array for ultrasound/photoacoustic dual modality imaging," Opt Express 22(21), 26365-26374 (2014).

[14] M. Jaeger et al., "Fourier reconstruction in optoacoustic imaging using truncated regularized inverse k-space interpolation," Inverse Probl 23(6), S51-S63 (2007).

[15] R. G. Rayavarapu et al., "Synthesis and bioconjugation of gold nanoparticles as potential molecular probes for light-based imaging techniques," International journal of biomedical imaging 2007(29817 (2007). 